\title{
Acute Thrombocytopenia After Infusion of an Iodinated Contrast Medium: A Case Report
}

\author{
Giovanni Barillari and Samantha Pasca*
}

Center for Hemorrhagic and Thrombotic Diseases, University Hospital, Udine, Italy

\begin{abstract}
lodinated contrast medium, administered during several diagnostic tests may be frequent cause of anaphylactic reactions or rashes. Thrombocytopenia after injection of iodinated contrast medium is a most rare adverse event, but several published reports have shown that this complication is more common than previously thought. In patients with acute drug induced thrombocytopenia, antibody tests are often negative. Thrombocytopenia due to iodinated contrast medium is transient, but the patient is made sensitive to the drug; further examinations should therefore always be performed in the absence of iodinated contrast medium.
\end{abstract}

Keywords: Thrombocytopenia; Iodinated contrast medium; Platelet disorders

\section{Case Report}

Iodinated Contrast Medium (ICM), administered during several diagnostic tests such as angiography, angioCT or CT scan, may be frequent cause of anaphylactic reactions or rashes [1-3]. Thrombocytopenia after injection of ICM is a most rare adverse event, but some studies have reported it [4-8]. Several hypotheses have been suggested about the mechanism that induces this syndrome, but none was confirmed such as the real cause of thrombocytopenia. Patient with a history of drug-induced thrombocytopenia may show antibody tests negative [4-9]. We report a case of two subsequent episodes of severe thrombocytopenia due to ICM.

A 73-year-old man with bilateral carotid stenosis, severe right hip osteoarthritis and iliac atheromasia was admitted to our hospital for evaluation of Aortic Abdominal Aneurysm (AAA). At the time of hospitalization the medications were telmisartan associated with hydrochlorothiazide, rosuvastatin, indobufene and oxycodone. Laboratory data included a platelet count of $214 \times 10^{3} \mu \mathrm{L}$, INR and aPTT in range. The day after admission, the patient underwent an angioCT with a previous injection of $20 \mathrm{ml}$ iodinated contrast medium, immediately post ICM infusion the patient presented acute epigastric pain and mild cyanosis spontaneously resolved after few minutes. His platelet count was $28 \times 10^{3} \mu \mathrm{L}$, decreased to $7 \times 10^{3} \mu \mathrm{L}$ six hours later, but both, INR and aPTT, remained within normal limits. No major or minor bleedings were found. On the basis of these findings, the physicians excluded a Disseminated Intravascular Coagulation (DIC). No other causes of thrombocytopenia were evident: the patient was not treated with heparin and the other drugs, he assumed, could not be correlated with a decrease in platelets. Only the administration of the ICM could therefore have caused this acute thrombocytopenia. His platelet count increased spontaneously and reached $181 \times 10^{3} \mu \mathrm{L}$ by the fifth day after procedure. Subsequent checks showed that platelet value has always been within normal range.

Four years before, the patient had a similar episode of thrombocytopenia attributed to ICM infusion. Still in that case, the patient was admitted to hospital for a diagnostic evaluation of AAA. CT scan was performed with and without ICM infusion, but immediately after ICM injection the patient presented an abdominal pain, followed by a severe decrease of platelet count, from $208 \times 10^{3} \mu \mathrm{L}$ at admission, to $3 \times 10^{3} \mu \mathrm{L}$. His platelet count increased quickly after corticosteroid therapy and platelet infusion and remains within normal range, until the new recent event. Following this first episode, corticosteroids and anti-histamines were administered still in the last case, but they were not sufficient to prevent thrombocytopenia.

\section{Discussion}

Thrombocytopenia was generally considered a non common adverse event following injection of iodinated contrast medium, but several published reports have shown that this complication is more common than it was previously thought [4-7].

Our patient developed two episodes of abdominal pain and severe thrombocytopenia immediately after ICM injection. In the first case the patient was immediately treated with corticosteroids and transfused with platelets to prevent the risk of intracranial or intrapulmonary haemorrhage, the event was resolved within a short time. Following this previous experience, corticosteroids are still administered before the last radiographic procedure, but they were not sufficient to avoid acute thrombocytopenia. If thrombocytopenia is drug-induced, such in our case, there are no evidences about the real efficacy of corticosteroids [4-9]. In the past, few cases of platelet decrease due to disseminated intravascular coagulation after ICM injection have been reported [710], but in our case laboratory tests excluded it. The platelet count is in fact rapidly increased after discontinuation of the ICM infusion. The exact mechanism of acute thrombocytopenia due to contrast medium is still not entirely known. A rapid onset, followed by a proliferation of megakaryocytes in the marrow, indicate destruction or a consumption of peripheral platelets, these are some events that characterize the thrombocytopenia. Some drugs or chemical agents may cause an immune-mediated platelet destruction [7], but several hypotheses have been proposed such as mechanism that causes specific antibody induction: drug induced conformational change of surface glycoprotein IIb/IIIa, drug induced autoantibody reacting with autologous platelets,

*Corresponding author: Samantha Pasca, Center for Hemorrhagic and Thrombotic Diseases, University Hospital of Udine, P.le S. Maria della Misericordia 1533100 Udine, Italy, Tel: +39-0432-554488; Fax: +39-0432-552848; E-mail: sampasca@alice.it

Received October 03, 2012; Accepted October 23, 2012; Published October 25 2012

Citation: Barillari G, Pasca S (2012) Acute Thrombocytopenia After Infusion of an lodinated Contrast Medium: A Case Report. J Clin Case Rep 2:218. doi:10.4172/2165-7920.1000218

Copyright: () 2012 Barillari G, et al. This is an open-access article distributed under the terms of the Creative Commons Attribution License, which permits unrestricted use, distribution, and reproduction in any medium, provided the original author and source are credited. 
Citation: Barillari G, Pasca S (2012) Acute Thrombocytopenia After Infusion of an lodinated Contrast Medium: A Case Report. J Clin Case Rep 2:218. doi:10.4172/2165-7920.1000218

soluble drug that induces a binding of a drug induced antibody to membrane protein, formation of immune complex mediated by PF4, chemical agent and specific antibody [4]. In patients with acute drug induced thrombocytopenia, antibody tests are often negative [4-9].

Thrombocytopenia due to iodinated contrast medium is transient, but the patient is made sensitive to the drug; further examinations should therefore always be performed in the absence of ICM.

\section{Disclosures}

The authors declare no conflict of interest relative to this paper.

\section{References}

1. Singh J, Daftary A (2008) lodinated contrast media and their adverse reactions. J Nucl Med Technol 36: 69-74.

2. Dewachter P, Laroche D, Mouton-Faivre C, Bloch-Morot E, Cercueil JP, et al. (2011) Immediate reactions following iodinated contrast media injection: a study of 38 cases. Eur J Radiol 77: 495-501.

3. Scherer K, Harr T, Bach S, Bircher AJ (2010) The role of iodine in hypersensitivity reactions to radio contrast media. Clin Exp Allergy 40: 468-475.

4. Garcia Bueno MJ (2009) Thrombocytopenia and iodinated contrast. J Investig Allergol Clin Immunol 19: 511-512.

5. Kudoh Y, Kijima T, limura O (1991) Acute thrombocytopenia after intravenous infusion of radiographic contrast medium. Nihon Jinzo Gakkai Shi 33: 885-888.

6. Chang JC, Lee D, Gross HM (1989) Acute thrombocytopenia after i.v. administration of a radiographic contrast medium. AJR Am J Roentgenol 152 947-949.

7. Lacy J, Bober-Sorcinelli KE, Farber LR, Glickman MG (1986) Acute thrombocytopenia induced by parenteral radiographic contrast medium. AJR Am J Roentgenol 146: 1298-1299.

8. Shojania AM (1985) Immune-mediated thrombocytopenia due to an iodinated contrast medium (diatrizoate). Can Med Assoc J 133: 123.

9. Aster RH, Bougie DW (2007) Drug-induced immune thrombocytopenia. N Eng J Med 357: 580-587.

10. Lalli AF (1980) Contrast media reactions: data analysis and hypothesis Radiology 134: 1-12. 